Journal of Computer Science 8 (9): 1428-1433, 2012

ISSN 1549-3636

(C) 2012 Science Publications

\title{
Principal Component Analysis Based Feature Extraction, Morphological Edge Detection and Localization for Fast Iris Recognition
}

\author{
${ }^{1}$ Suganthy, M. and ${ }^{2}$ P. Ramamoorthy \\ ${ }^{1}$ School of Electronics and Communication, Veltech Engineering College, Chennai, India \\ ${ }^{2}$ Electronics and Communication Sri Sakthy Institute of Engineering and Technology, Coimbatore, India
}

\begin{abstract}
This study involves the Iris Localization based on morphological or set theory which is well in shape detection. Principal Component Analysis (PCA) is used for preprocessing, in which the removal of redundant and unwanted data is done. Applications such as Median Filtering and Adaptive thresholding are used for handling the variations in lighting and noise. Features are extracted using Wavelet Packet Transform (WPT). Finally matching is performed using KNN. The proposed method is better than the previous method and is proved by the results of different parameters. The testing of the proposed algorithm was done using CASIA iris database (V1.0) and (V3.0).
\end{abstract}

Key words: Principal Component Analysis (PCA), Morphological, Wavelet Packet Transform (WPT), K Nearest Neighbor (KNN)

\section{INTRODUCTION}

It is a complicated and a minute work to develop the automated authentication of individuals. At present the methods such as passwords and electronic cards are not secure and are prone to theft and misuse. A number of Authentication technologies such as retinal, fingerprint, hand geometry, facial and voice, iris systems have been developed due to our increasing knowledge of the human body's physiology. Biometric identification techniques use inherent physical or behavioral characteristics which are unique among all individuals. Generally used biometrics traits are fingerprint, face, palm print, iris, retina, ear, DNA, signature, voice, keystroke, gait.

Iris is an important feature of the human body among the physiological biometrics and it is unique and has better stability. Now a days, Iris recognition technology has more advantage in the field of information security and verification of individuals in the areas such as controlling access to security zones, verification of passengers at airports, stations, computer access at defense establishments, research organization, data base access control in distributed systems. Iris recognition systems are currently being deployed in many countries for airline crews, airport staffs, national ID cards and missing children identification

The round contractile membrane of the eye suspended between cornea and lens is termed as the Iris and it is darkened in the shape of circle by the pupil. Iris begins to form during gestation and by the eighth month of the pregnancy it gets completely formed. The very special nature of the iris pattern gains from the details of the texture like cornea, crypts, filaments, flecks, radial furrows, stripes, arching ligaments. These irregularly shaped microstructures are so randomly distributed patterns which prove the human iris as one of the most important and reliable biometric traits.

An Iris is enclosed by two concentric circles. The inner circle is lying between the iris and the pupil and the outer circle is lying between iris and sclera. These circles are characterized by gray scale changes. The inner and outer boundaries look like circles but they are not concentric. The eyelids and eyelashes hide the clear appearance of the both circles.

The parts of the iris are formed by many fibers, contraction furrows, coronas, freckles, rifts, crypts and pits. Figure 1 depicts the parts of iris (Meng and $\mathrm{Xu}$, 2006). These features and their special relationship to each other provide abundant information, which is useful while authenticating a person through iris recognition system.

The fingerprint identification is the mature biometric technique used for criminal investigations. Major representations of the finger are based on the entire image, finger ridges, or salient features derived from the ridges (minutiae). These characteristics are used to generate an orientation field of the fingerprint, which subsequently provides the discriminating details for authentication of persons. Their low cost and minimum and less storage capacity are favorable. Authentication by hand geometry has gained widespread interest. The feature vector incorporates information concerning the length of the fingers and hand shapes. Their drawbacks concern their relatively high False Accept Rates (FAR) and False Reject Rates (FRR).

Corresponding Author: Suganthy, M., School of Electronics and Communication, Veltech Engineering College, Chennai, India 


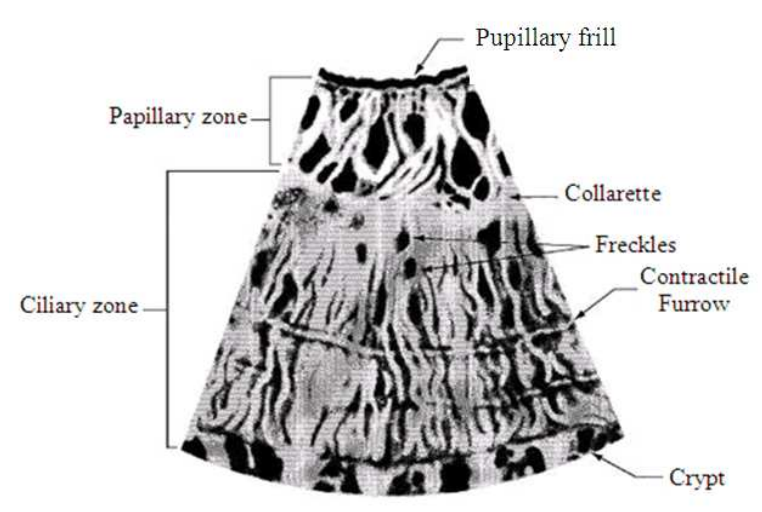

Fig. 1: Structure of an iris

Face is one of the most common methods of identification and also acquiring face images is nonintrusive. Two primary approaches used in face recognition are: (i) Transform approach ii) Attributebased approach. It is the most challenging job to improve face recognition techniques which can tolerate the effects of aging, facial expressions, slight variations in the imaging environment and variations in the pose of face with respect to camera. A general solution to these problems has yet to be presented. Voice is a characteristic of an individual. It lacks in uniqueness to permit identification of an individual from a large database Furthermore a voice signal is degraded in quality by the microphone, communication channel and digitizer characteristics. This system is also susceptible to a number of problems such as imposters, noise and physiological changes of the person.

It has low FRRs and FARs and the digital signature established is highly unique. Replication of the iris and fraudulent efforts are highly unlikely. We focused our attention on feature extraction methods which are explained in the succeeding section. Iris Pattern has low genotypic error rate and phenotypic error rate and the digital signature established is highly unique. Duplication of the iris and deceitful efforts are highly restricted. We focused our attention on feature extraction methods which are explained in the succeeding section.

Iris feature extraction methods overview: Feature extraction is a special form of dimensionality reduction and contains more information about the original image. The input data which is to be processed is transformed into a reduced representation set of features. This is referred as feature extraction.

Daughman, 2004; Hastie and Tibshirani, 1996, developed an algorithm for iris recogntion one and half decades before, (Daughman, 2004; Hastie and Tibshirani, 1996), still it remains valuable since it provides solutions for beginners those who are involved in research.

In (Wang and Mei, 2006), for contrast enhancement, the iris image is preprocessed. The data is obtained from the iris image by moving a ring mask through the entire image. By using this data the iris and pupil are reconstructed from the original image then the iris was cropped out from the reconstructed image using the centre and the radius of iris. The iris data (circular shape) is stretched into a rectangular shape which is known as normalization.

The method followed in (Lim, 1990) Wavelet Packet Transform (WPT) is used for texture extraction. Wavelet transform decompose the image into four subbands of detail coefficients, namely approximation, horizontal, vertical and diagonal. Haar wavelet is used for our work, after the transformation the sub images are selected for encoding.

Principal Component Analysis (PCA) (Rafael and Woods, 2002) is a well-known and one of the most successful techniques used in image recognition and compression for extracting feature and representing data. It is technique widely used in the area of pattern recognition, computer vision and signal processing. The purpose of PCA is to reduce the large dimensionality of the data space (observed variables) to the smaller intrinsic dimensionality of feature space (independent variables), which are needed to describe the data economically. This is the case when there is a strong correlation between observed variables. By discarding minor components, the PCA effectively reduces the number of features and displays the data set in a lowdimensional subspace. In this study the feature extraction algorithm based on PCA is chosen. The coefficients of these methods are used as feature vectors which efficiently represent iris signals.

In an eye image due to the reflection from the illumination source, bright spot is formed in the pupil. Global threshold cannot be applied for pupil circle detection by replacing the pixel by the value of the neighborhood pixel inside the pupil because it almost fills the circle. In order to overcome this problem, the captured image is passed through the median filter.

Figure 2 shows the steps involved in the proposed method.

In the operation of median filter, a 2-D mask is applied to each pixel in the input image. To apply the mask means to centre it in a pixel, evaluating the covered pixel brightness and determining which brightness value is the median value. 


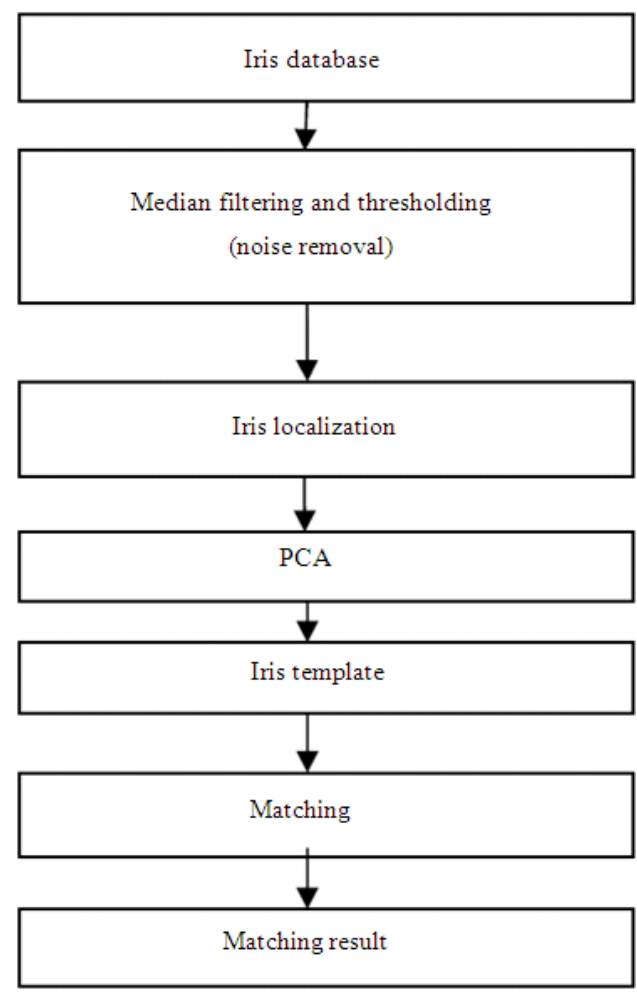

Fig. 2: Block diagram
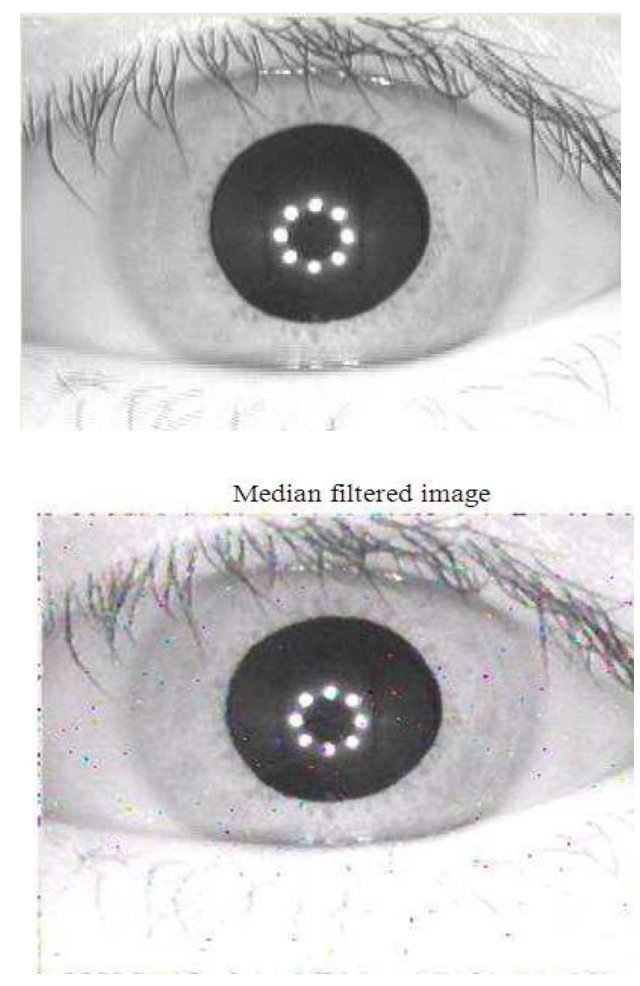

Fig. 3: Image before and after a median Filter
The median value is determined by placing the brightness values in ascending order and selecting the centre value. The obtained median value will be the value for that pixel in the output image.

For example, the pixel values within a slide or mask are 5, 6, 7 50, 9, 10 and 15 and the pixel being processed has a value of 55 . The output of the median filter the current pixel location is 9 , which is the median of the five values. Like low pass filtering, median filtering smoothens the image and is thus useful in reducing noise. In our work detected edge is enhanced using median filer.

Median filter can preserve discontinuities in a step function and perform smoothening of pixels without affecting the other pixels. (Liam et al., 2002). The output of median filter is given in Fig. 3.

Thresholding: Image thresholding is used for extracting the significant or desired part of an image and removing the unwanted part or noise. The point operator of major interest is called thresholding which selects pixels that have a particular value, or that are within a specified range. With thresholding, the image can be segmented based on the colour. This holds true under the assumption that a reasonable threshold value is chosen. A reasonable threshold value is the taken from the histogram of the original image. The point operator helps us to find objects in a picture if the brightness level or range is known. Hence the object's brightness must be known.

There are two main forms: (1) Uniform and (2) Adaptive thresholding. In uniform thresholding, pixels above a specified level are set to white; those below the specified level are set to black. Adaptive thresholding is when a different threshold is used for different regions in the image. This may also be known as local or dynamic thresholding the thresholded image is shown in Fig. 4.

Morphology based iris localization: The intensity of iris is close to sclera and is obscured by eyelashes and eyelids which make it easy to locate. Similarly, the size and uniform dark shade make the pupil to be identified easily. Hence, the pupil and iris are approximated to be concentric which enhances a reliable entry point for auto detection, an iris region between the pupil and the sclera from an eye is obtained by iris localization. The iris region approximation includes two circles: one for the iris/sclera boundary and the other for the iris/pupil boundary.

The estimation efficiency of the pupil depends on Computational speed rather than accuracy since it is simple in shape and is the darkest region in an eye image and can be extracted using a suitable threshold.

The centre and radius of the pupil after removal of eyelashes and eyelids can be exactly obtained using Morphological process. 


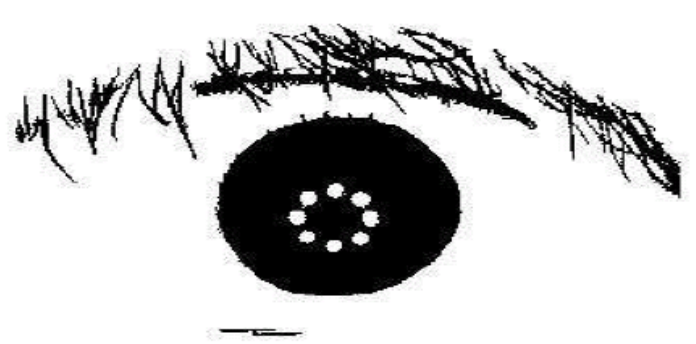

Fig. 4: Image after thresholding

Hence this concept is found to be very useful for image processing applications which include segmentation, denoising, shape detection and description of objects.

Dilation and erosion: Dilation and erosion are basic morphological operations. They are defined in terms of more elementary set operations, but are employed as the basic elements of many algorithms. Dilation is used to increase the object size where as erosion is used to diminish the size of the object. Both dilation and erosion are produced by the moving the mask around the image. The mask which is also called as structuring element or subimage or kernel (Lim, 1990) and (Soille, 2004) and it has both a shape and an origin. The following Eq. 1 and 2 are generally used for dilation and erosion:

Dilation $=\mathrm{X} \oplus \mathrm{S}$

Erosion $=\mathrm{X} \oplus \mathrm{S}$

where, $\mathrm{X}$ is a orinal image and $\mathrm{S}$ is a srructuring element.

Based on the image which is to be dilated or eroded the size of the structuring element is choosed.it is a odd square matrix which containts binary elements that is 0 's and 1's and also the dimension should not exceed the size of the image which is to be processed. The structuring element is a set of coordinates. The origin of the structuring element is the centre element for most cases but it may not be centre point for less cases. The structuring element (Lim, 1990; Soille, 2004) is used to remove the eyelashes and eyelids whose dimension based on region of interest. Following are the examples of structuring elements. In our work we choosed the $3 \times 3$ structuring element having its all element as 1 :

$\begin{array}{lllllllll}1 & 1 & 1 & 0 & 1 & 0 & 1 & 1 & 0 \\ 1 & 1 & 1 & 1 & 1 & 1 & 1 & 0 & 1 \\ 1 & 1 & 1 & 0 & 1 & 0 & 1 & 0 & 1\end{array}$

The origin of the structuring element identifies the pixel of interest in the input eye image and all the pixels in its neighborhood is assigned to the corresponding pixel in the output image. The result of Erosion is subtracted from the result of dilation will provide the edge. The following Eq. 3 and 4 are used for dilation and erosion. The result of erosion and dilation is given in Fig. $5 \mathrm{a}$ and $\mathrm{b}$ :

$$
\begin{aligned}
& F \theta B={ }_{B(i, j)}[F(x+i, y+j)-B(i, j)+1] \\
& F \oplus B=B_{(i, j)}^{\vee}[F(x-i, y-j)+B(i, j)-1]
\end{aligned}
$$

Principal Components Analysis PCA: Linear Discriminant Analysis (LDA), Independent Component Analysis and PCA are some of the techniques used for feature extraction, among them PCA is powerful method in image formation, Data patterns, similarities and differences between them are identified efficiently. The other main advantage of PCA is dimension will be reduced by avoiding redundant information, (Daugman, 1993) without much loss. Better understanding of principal component analysis is through statistics and some of the mathematical techniques which are Eigen values, Eigen vectors. PCA is a useful statistical and common technique that has found application in fields such as image recognition and compression.

Principal
$\begin{gathered}\text { Component } \\ \text { procedure }\end{gathered}$ $\begin{gathered}\text { Analysis } \\ \text { that }\end{gathered}$ (PCA) is a Transformations to map data from high dimensional space to low dimensional space. The low dimensional space can be determined by Eigen vectors of the covariance matrix.

\section{The steps involved in PCA include:}

- The mean valve $\overline{\mathrm{S}}$ of the given data set" $S$ " is found

- Subtract the mean value say from S. from these valves a new matrix is obtained. Let say "A"

- Covariance is obtained from the matrix i.e., $\mathrm{C}=$ $\mathrm{AA}^{\mathrm{T}}$ Eigen values are obtained from the covariance matrixes that are $\mathrm{V}_{1} \mathrm{~V}_{2} \mathrm{~V}_{3} \mathrm{~V}_{4} \ldots \mathrm{V}_{\mathrm{N}}$,

- Finaly Eigen vectors are calculated for covariance matrix $\mathrm{C}$

- Any vector $\mathrm{S}$ or $\mathrm{S}-\overline{\mathrm{S}}$ can be written as linear combination of eigen vectors shown in Eq. 5

- Because covariance matrix is symmetric it form basis $\mathrm{V}_{1} \mathrm{~V}_{2} \mathrm{~V}_{3} \mathrm{~V}_{4} \ldots$

$$
\mathrm{V}_{\mathrm{N}} \mathrm{S}-\overline{\mathrm{S}}=\mathrm{b}_{1} \mathrm{u}_{1}+\mathrm{b}_{2} \mathrm{u}_{2}+\mathrm{b}_{3} \mathrm{u}_{3}+\ldots+\mathrm{b}_{\mathrm{N}} \mathrm{u}_{\mathrm{N}}
$$

- $\quad$ Only Largest eigen values are kept to form lower dimension data set (Eq. 6):

$\hat{\mathrm{S}}-\overline{\mathrm{S}}=\sum_{\mathrm{i}=0}^{1} \mathrm{~b}_{1} \mathrm{u}_{1} ; 1<\mathrm{N}$ 


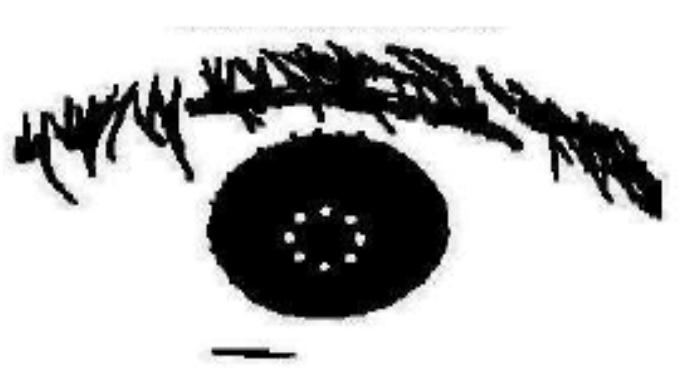

(a)

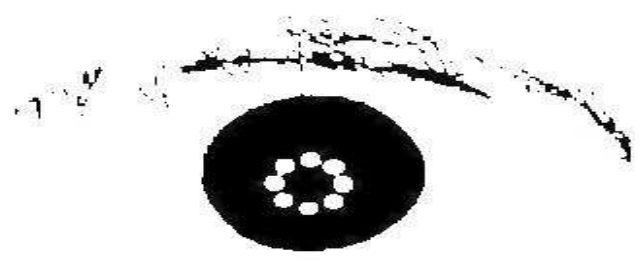

(b)

Fig. 5: Comparison of different method (a) Eroded Image (b) Dilated image

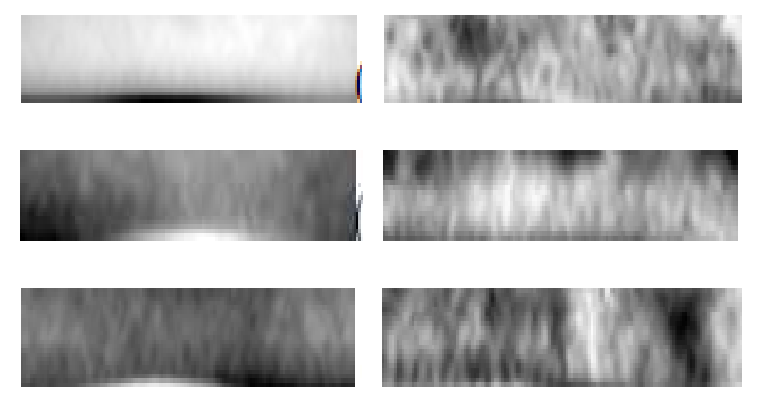

Fig. 6: Obtained principal components

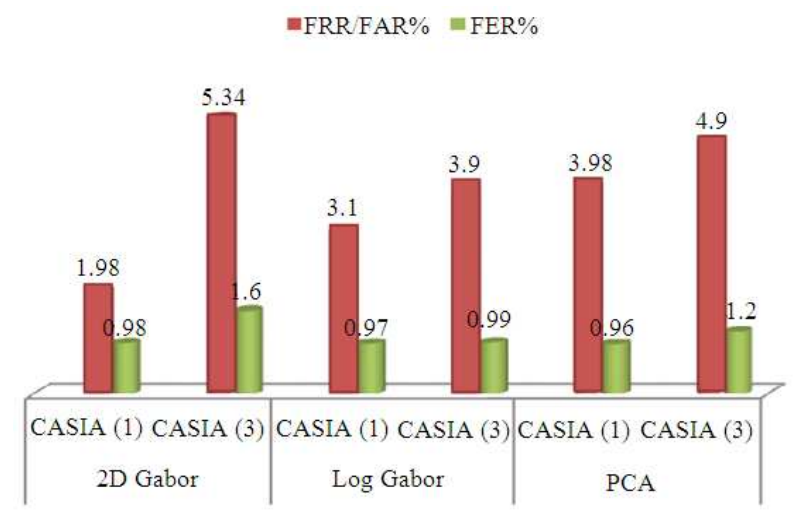

Fig. 7: Performance of different methods

The components in lower dimension space are called principal components which are guaranteed to be independent (Daugman, 1993) only if the data set is jointly normally distributed. PCA is sensitive to the relative scaling of the original variables. Depending on the field of application, it is also named as discrete Karhunen-Loève Transform (KLT), or the Hotelling transform. The principal components obtained are shown in the following Fig. 6.

The signature of each image is found by multiplying the transpose of zero mean vectors with feature vectors.

The comparison of different parameters for different methods is shown in Fig. 7.

Matching: The features of the test image are compared with the features of images in the database. K-Nearest Neighbors (KNN): It is used for classifying the objects in feature face based on the training sample chosen. It is simplest learning algorithm among the all, the $\mathrm{k}$ value depends upon the data, if it is large noise effects will be lessen but boundaries exist between classes which are less distinct. If $\mathrm{K}=1$ it is known as $\mathrm{KNN}$ algorithm. Computation of $\mathrm{K}$ involves the following steps:

- $\quad$ The parameter $\mathrm{K}$ = number of nearest neighbors on hand the value is determined by us.

- The distance between the query-instance and all the training samples is found using any distant algorithm. City block (Domeniconi et al., 2002) distance arameter in KNN is used for matching and is given in Eq. 7:

$\mathrm{d}(\mathrm{a}, \mathrm{b})=\sum_{\mathrm{i}=1}^{\mathrm{n}} \mid \mathrm{bi}-\mathrm{ai}$

- Distances for all the training samples will be sorted out and the nearest neighbour based on the K-th minimum distance is taken.

- The majority of nearest neighbours is taken as the prediction value.

It involves:

- Training phase in which the feature vectors are stored and the class labels are assigned to the training samples

- Classification phase in which the test sample is classified by assigning (Hastie and Tibshirani, 1996) the label which is most frequently occurring among the $\mathrm{k}$ training samples closest to the test sample. City block (Domeniconi et al., 2002) distance parameter in $\mathrm{KNN}$ is used for matching and is given in Eq. 8: 


$$
d(a, b)=\sum_{i=1}^{n} \mid b i-a i
$$

Experimental result: The general performance metrics such as False Accept Rate (FAR), False Reject Rate (FRR) are choose for performance analysis of proposed systems. We compared the recognition rate in Table 1 Wavelet Packet Transform (WPT) feature extraction method with proposed method which involved PCA. The experiment result indicates that recognition rate achieves $98 \%$ while 5 images among 10 images in each class are used as the training sample which is shown in table. If the testing samples are increased, the recognition rate will improve, but the training time will be also greatly prolonged. The performance evaluation of different methods for PCA components is done, the Fig. 8 depicted this.

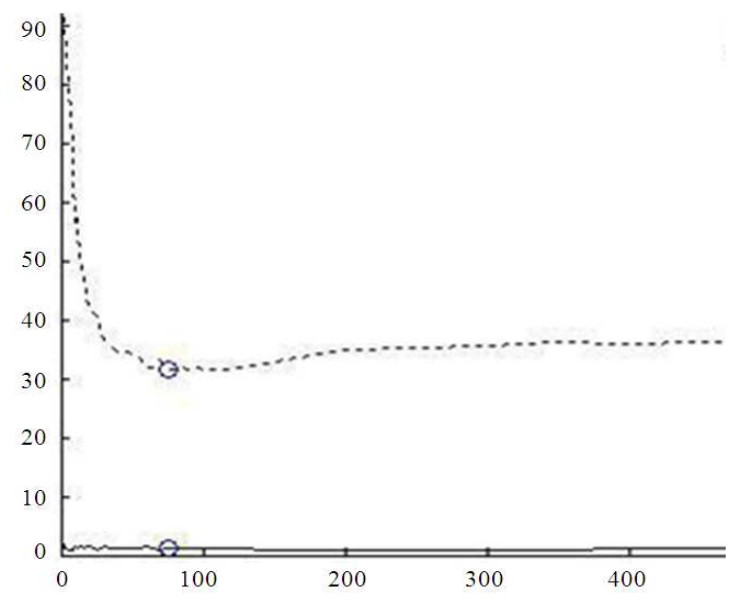

Fig. 8: PCA Performance Evaluation

$\underline{\text { Table 1: Recognition rate of two methods in different testing samples }}$

\begin{tabular}{lll}
\hline & Recognition rate & \\
& - & \\
Number of testing samples & PCA & WPT with WPT \\
\hline 5 & 0.9143 & 0.9446 \\
7 & 0.9388 & 0.9530 \\
8 & 0.9400 & 0.9757 \\
10 & 0.9486 & 0.9772 \\
15 & 0.9500 & 0.9800 \\
\hline
\end{tabular}

\section{CONCLUSION}

This algorithm adopted morphological operation for the inner and outer iris edge detecting and locating and has the merits of short localization time and high localization precision. The experimental results indicate that the proposed algorithm is a kind of effective iris locating algorithm in the aspect of time consumed and localization accuracy. Future work includes different matching algorithm and feature extraction method for novel iris recognition which will give better result.

\section{REFERENCES}

Daughman, J., 2004. How iris recognition works. IEEE Trans. Circ. Syst. Video Technol., 14: 21-30.

Daugman, J.G., 1993. High confidence visual recognition of persons by a test of statistical independence. IEEE Trans. Patt. Anal. Mach. Intell., 15: 1148-1161. DOI: 10.1109/34.244676

Domeniconi, C., P. Jing and D. Gunopulos, 2002. Locally adaptive metric nearest-neighbor classification. IEEE Trans. Patt. Anal. Mach. Intell., 24: 1281-1285. DOI: 10.1109/TPAMI.2002.1033219

Hastie, T. and R. Tibshirani, 1996. Discriminant adaptive nearest neighbor classification. IEEE Trans. Patt. Anal. Mach. Intell., 18: 607-616. DOI: 10.1109/34.506411

Liam, L.W., A. Chekima, L.C. Fan and J.A. Dargham, 2002. Iris recognition using self-organizing neural network. Proceedings of the Student Conference on Research and Development, Jul. 16-17, IEEE Xplore Press, Malaysia, pp: 169-172. DOI: 10.1109/SCORED.2002.1033084

Lim, J.S., 1990. Two-Dimensional Signal and Image Processing. 1st Edn., Prentice Hall, New Jeresy, ISBN-10: 0139353224 pp: 694.

Meng, H. and C. Xu, 2006. Iris recognition algorithms based on Gabor wavelet transforms. Proceedings of the IEEE International Conference on Mechatronics and Automation, Jun. 25-28, IEEE Xplore Press, pp: 1785-1789. DOI: 10.1109/ICMA.2006.257485

Rafael, C.G. and R.E. Woods, 2002. Digital Image Processing. 2nd Edn., Prentice Hall, New Jeresy.

Soille, P., 2004. Morphological Image Analysis. 2nd Edn., Springer, New York, pp: 391.

Wang, J. and X. Mei, 2006. Iris feature extraction based on wavelet packet analysis. Proceedings of the International Conference on Communications, Circuits and Systems, Jun. 25-28, IEEE Xplore Press, Chengdu, pp: 31-34. DOI: 10.1109/ICCCAS.2006.284579 